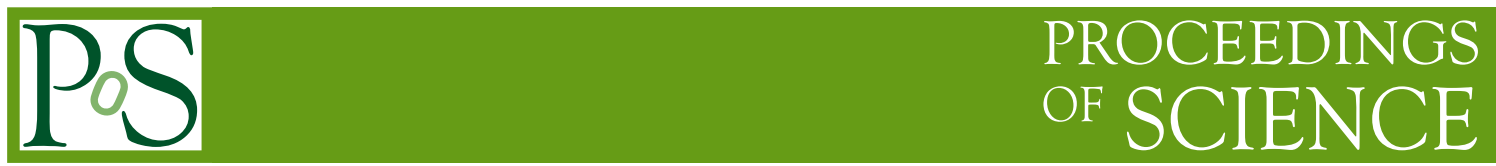

MPP-2010-176

\title{
NLO corrections to WWZ and ZZZ production at the ILC
}

\author{
Fawzi Boudjema \\ LAPTH, Université de Savoie, CNRS, \\ BP110, F-74941 Annecy-le-Vieux Cedex, France \\ E-mail: boudjema@lapp.in2p3.fr

\section{Le Duc Ninh*, Marcus M. Weber} \\ Max-Planck-Institut für Physik (Werner-Heisenberg-Institut), \\ D-80805München, Germany \\ E-mail: leducninh@gmail.com, mmweber@mppmu.mpg.de
}

\section{Sun Hao}

Department of Physics, State University of NewYork, Buffalo, NY14260, USA

E-mail: hsun6@buffalo.edu

We calculate the full one-loop electroweak corrections to tri-boson production (ZZZ and WWZ) at the ILC. This is important to understand the Standard Model (SM) gauge quartic couplings which can be a window on the mechanism of spontaneous symmetry breaking. We find that even after subtracting the leading QED corrections, the electroweak corrections can still be large especially as the energy increases.

3rd Computational Particle Physics Workshop

September 23-25, 2010

KEK Tsukuba Japan

\footnotetext{
${ }^{*}$ Speaker.
} 


\section{Introduction}

Due to its clean environment an $e^{+} e^{-}$linear collider in the TeV range is an ideal machine to probe in detail and with precision the inner working of the electroweak structure, in particular the mechansim of symmetry breaking. From this perspective the study of $e^{+} e^{-} \rightarrow W^{+} W^{-} Z$ and $e^{+} e^{-} \rightarrow Z Z Z$ may be very instructive and would play a role similar to $e^{+} e^{-} \rightarrow W^{+} W^{-}$at lower energies. Indeed it has been stressed that $e^{+} e^{-} \rightarrow W^{+} W^{-} Z$ and $e^{+} e^{-} \rightarrow Z Z Z$ are prime processes for probing the quartic vector boson couplings [1]. In particular deviations from the gauge value in the quartic $W^{+} W^{-} Z Z$ and $Z Z Z Z$ couplings that are accessible in these reactions might be the residual effect of physics intimately related to electroweak symmetry breaking. Since these effects can be small and subtle, knowing these cross sections with high precision is mandatory. This calls for theoretical predictions taking into account loop corrections.

In this report we discuss the next-to-leading order (NLO) corrections to the processes $e^{+} e^{-} \rightarrow$ ZZZ and $e^{+} e^{-} \rightarrow W^{+} W^{-} Z$ at the future international linear collider (ILC). We also address some technical issues related to numerically stable evaluation of one-loop integrals, which is a challenge for one-loop multi-leg automatic calculations.

\section{Tree level}

(a)

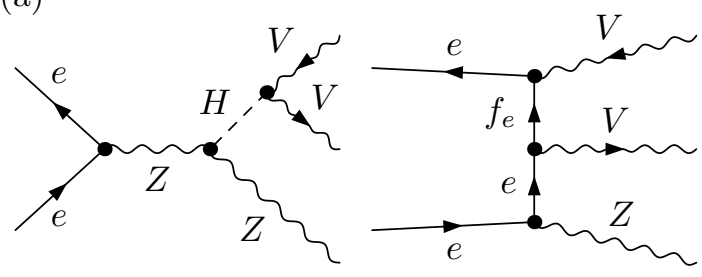

(b)

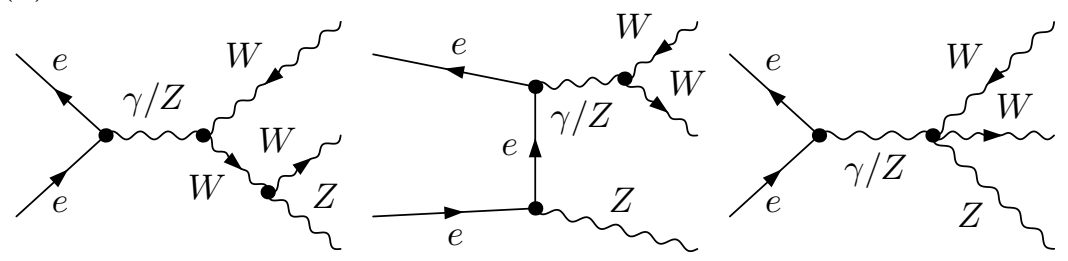

Figure 1: Representative Born diagrams for $e^{+} e^{-} \rightarrow Z Z Z$ and $e^{+} e^{-} \rightarrow W^{+} W^{-} Z$. Diagrams (a) contribute to both processes while diagrams of type $(b)$ contribute only to $e^{+} e^{-} \rightarrow W^{+} W^{-} Z$. The first diagram of type (a) will be referred to as the Higgsstrahlung contribution.

At leading order $W^{+} W^{-} Z$ and $Z Z Z$ final states are produced through the diagrams shown in Fig. 1. The important gauge couplings fermion-fermion-vector, $\gamma W W$ and $Z W W$, which also appear in the well-tested $e^{+} e^{-} \rightarrow W^{+} W^{-}$process, have been measured and found in good agreement with the prediction of the SM $[2,3]$. Both processes include the Higgsstrahlung contribution where the splitting $H^{\star} \rightarrow V V$ occurs. This contribution is small and vanishes in the large Higgs mass limit. Since the precision electroweak data suggest a Higgs mass below the $W W$ threshold, we restrict 
our study to the region $M_{H}<160 \mathrm{GeV}$. This means that the Higgsstrahlung contribution can not be resonant and therefore in our calculation no width is introduced. The important features at tree level are that the neutral quartic gauge coupling $Z Z Z Z$ vanishes in the SM and the charged $\gamma Z W W$, $Z Z W W$ couplings occur in the $e^{+} e^{-} \rightarrow W^{+} W^{-} Z$ process. In practice, the coupling $\gamma Z W W$ (and also $\gamma \gamma W W$ ) can be tested at lower energies via the process $e^{+} e^{-} \rightarrow W^{+} W^{-} \gamma$.

\section{NLO calculations}

Our calculations are done in the framework of the SM. The virtual corrections have been evaluated using a conventional Feynman-diagram based approach using standard techniques of tensor reduction. We use the packages FeynArts and FormCalc- 6.0 to generate all Feynman diagrams and helicity amplitude expressions [4]. We also use Sloops to check the correctness of the amplitudes by checking non-linear gauge invariance (see [5] and references therein). The total number of diagrams in the 't Hooft-Feynman gauge is about 2700 including 109 pentagon diagrams for $e^{+} e^{-} \rightarrow W^{+} W^{-} Z$ and about 1800 including 64 pentagons for $e^{+} e^{-} \rightarrow Z Z Z$. This already shows that $e^{+} e^{-} \rightarrow W^{+} W^{-} Z$ with as many as 109 pentagons is more challenging than $e^{+} e^{-} \rightarrow Z Z Z$. Indeed getting stable results for all scalar and tensor (up to rank 4) box integrals in the process $e^{+} e^{-} \rightarrow W^{+} W^{-} Z$ is a highly nontrivial task. The five-point integrals are reduced to four-point integrals by using the method of Denner-Dittmaier [6] which does not involve the Gram determinant, $\operatorname{det} G=\operatorname{det}\left(2 p_{i} \cdot p_{j}\right)$ with $p_{i, j}$ being the external momenta, in the denominator. The four-point (and three-point) tensor integrals are in turn recursively reduced to scalar integrals by using Passarino-Veltman (PV) method. The problem with this method is that the numerical results become unstable when the Gram determinant is small. For instance, the result for a rank-4 box integral includes tensor coefficients of the form

$$
D_{i j k l}=\frac{N(p, m)}{(\operatorname{det} G)^{4}}
$$

where the numerator is a complicated function of internal masses and external momenta whose indices have been excluded for simplicity. In many cases the function $N$ vanishes in the limit $\operatorname{det} G \rightarrow 0$, leaving the tensor coefficients finite. In particular, this is usually true if the internal particles are massive. This non-trivial behavior of the numerator which is a linear combination of scalar integrals can be spoiled by numerical cancellation or inconsistent approximations (like small mass/momentum approximations) in calculation of the scalar integrals, leading to numerical instabilities in the right-hand side of Eq. (3.1) when $\operatorname{det} G$ becomes small. A good way to solve this problem is therefore using higher-precision arithmetic in the calculation of loop integrals when numerical cancellation occurs.

It is important to notice that the PV method fails when $\operatorname{det} G$ is exactly zero. If this happens the $N$-point function of rank $M$ can be written as a combination of $(N-1)$-point functions of rank $M$. This is called segmentation [7]. We have exploited this fact to avoid the small $\operatorname{det} G \operatorname{region}$ by using segmentation if the following condition is met

$$
\frac{\operatorname{det}(G)}{\left(2 p_{\max }^{2}\right)^{3}}<10^{-7}
$$




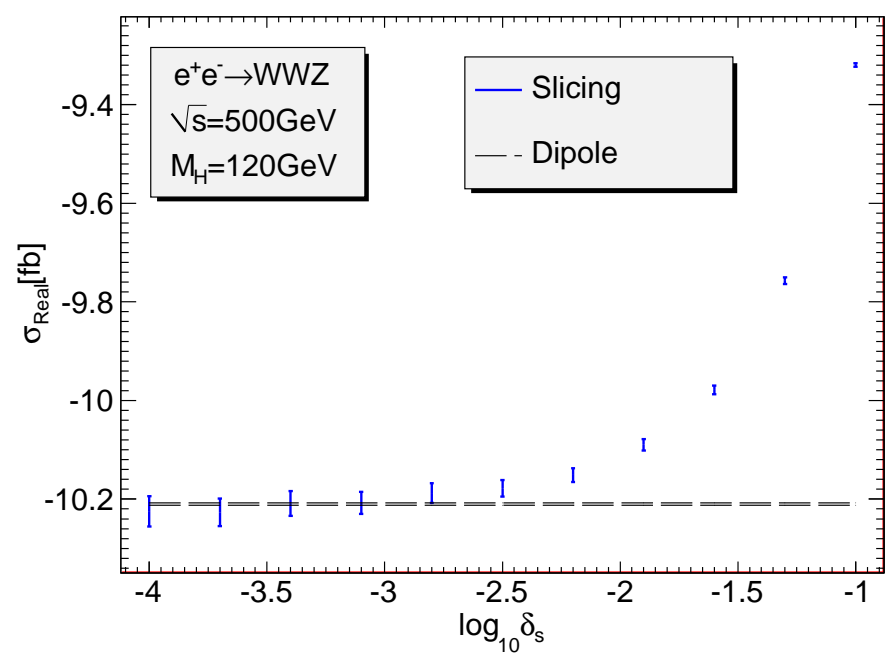

Figure 2: Dependence of $\sigma_{\text {real }}^{e^{+} e^{-} \rightarrow W^{+} W^{-} Z \gamma}$ on the soft cutoff $\delta_{s}\left(E_{\gamma}<\delta_{s} \sqrt{s} / 2\right)$ in phase-space slicing. Only the non-singular part is shown, i.e. the IR singular $\ln \left(m_{\gamma}^{2}\right)$ terms are set to zero. The result using dipole subtraction is shown for comparison with the error given by the width of the band.

where $p_{\max }^{2}$ is the maximum external mass of a box diagram. This extrapolation is used only for the four-point integrals and turns out to be good enough for the present calculations. We have compared this to the method of using higher precision arithmetic (quadruple precision for the Fortran 77 code) and obtained good agreement.

In addition to the virtual corrections we also have to consider real photon emission, i.e. the processes $e^{+} e^{-} \rightarrow W^{+} W^{-} Z \gamma$ and $e^{+} e^{-} \rightarrow Z Z Z \gamma$. The corresponding amplitudes are divergent in the soft and collinear limits. The soft singularities cancel against the ones in the virtual corrections while the collinear singularities are regularized by the physical electron mass. To extract the singularities from the real corrections and combine them with the virtual contribution we apply both the dipole subtraction scheme and a phase space slicing method. The former is used to produce the final results since it yields smaller integration errors as shown in Fig. 2. Further details are given in [8].

It is well-known that the collinear QED correction related to initial state radiation in $e^{+} e^{-}$processes is large. In order to see the effect of the weak corrections, one should separate this large QED correction from the full result. It means that we can define the weak correction as an infrared and collinear finite quantity. The definition we adopt in this paper is based on the dipole subtraction formalism. In this approach, the sum of the virtual and the so-called "endpoint" (see [9] for the definition) contributions satisfies the above conditions and can be chosen as a definition for the weak correction

$$
\sigma_{\text {weak }}=\sigma_{\text {virt }}+\sigma_{\text {endpoint }}
$$

For the numerical results shown in the next section, we will make use of this definition.

Before presenting our numerical results it is stressed that we have performed the calculation in at least two independent ways both for the virtual and the real corrections leading to two independent numerical codes (one code is written in Fortran 77, the other in $\mathrm{C}++$ ). A comparison 
of both codes has shown full agreement at the level of the integrated cross sections as well as all the distributions that we have studied. Moreover, we have done detailed comparisons with other groups $[10,11]$ and obtained good agreement.

\section{Numerical results}

To absorb large corrections to the electromagnetic coupling and universal corrections due to the isospin breaking effects we use

$$
\begin{aligned}
\alpha & =\alpha_{G_{\mu}}=\frac{\sqrt{2} G_{\mu} M_{W}^{2}}{\pi} \sin ^{2} \theta_{W}, \\
\alpha_{G_{\mu}} & =\alpha(0)(1+\Delta r)
\end{aligned}
$$

with $G_{\mu}$ denoting the Fermi constant and $\theta_{W}$ is the weak-mixing angle, at tree level. The explicit form of $\Delta r$ at one-loop order together with all the input parameters are given in [8]. When calculating the NLO corrections we have to subtract the one-loop $\Delta r$ contribution to avoid double counting. Since the real photon corrections are proportional to $\alpha(0)$, we require the full NLO corrections to be of order $\mathscr{O}\left(\alpha_{G_{\mu}}^{3} \alpha(0)\right)$.
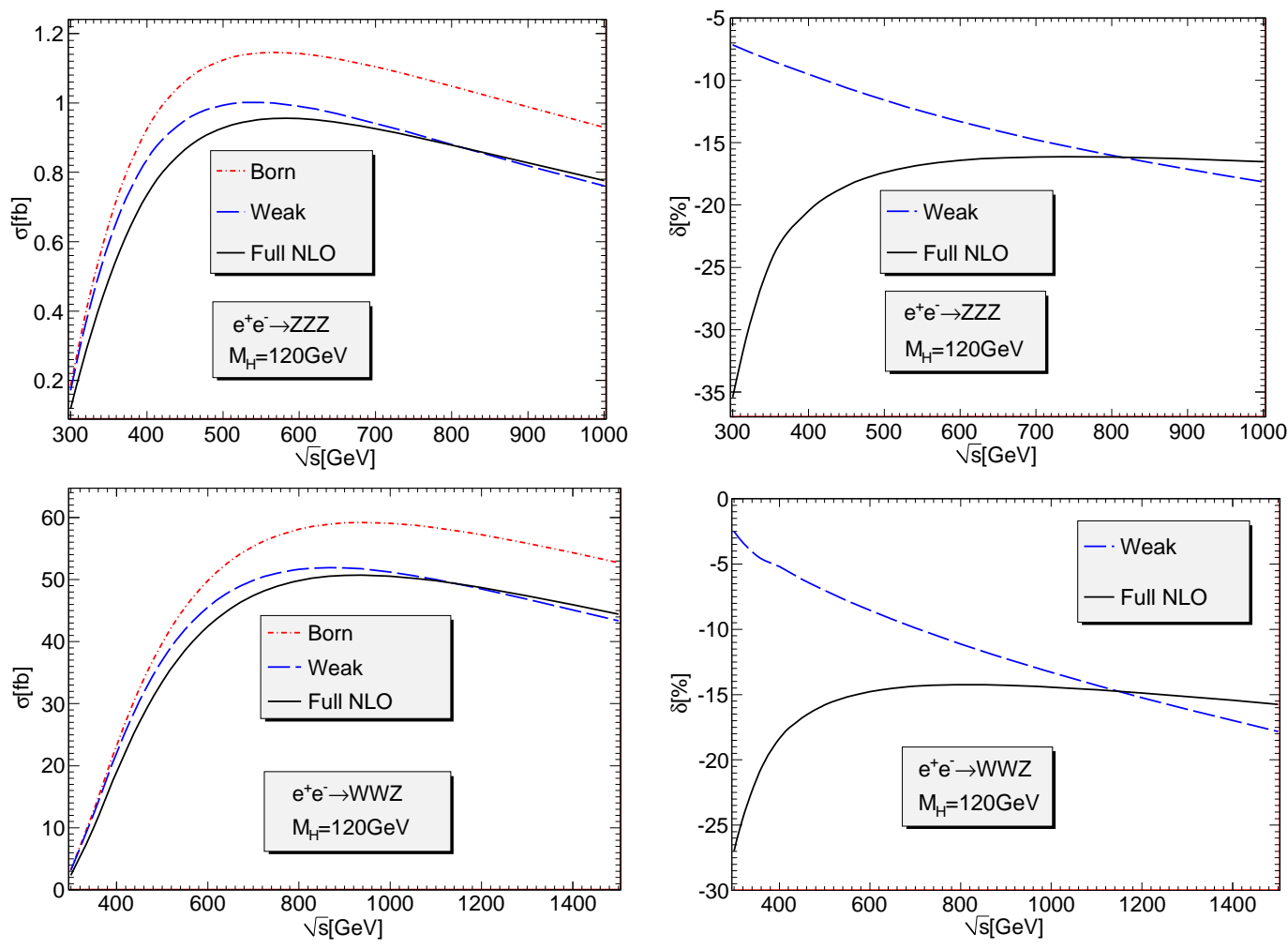

Figure 3: The total cross section for $e^{+} e^{-} \rightarrow Z Z Z$ (top) and $e^{+} e^{-} \rightarrow W^{+} W^{-} Z$ (bottom) as a function of $\sqrt{s}$ for the Born, full $\mathscr{O}(\alpha)$ and genuine weak correction. The panels on the left show the Born, the full NLO and the weak correction. The panels on the right show the corresponding relative (to the Born) percentage corrections. 
$\underline{e^{+} e^{-} \rightarrow Z Z Z:}$

As shown in Fig. 3 the tree-level cross section rises sharply once the threshold for production opens, reaches a peak of about $1.1 \mathrm{fb}$ around a centre-of-mass energy of $600 \mathrm{GeV}$ before very slowly decreasing with a value of about $0.9 \mathrm{fb}$ at $1 \mathrm{TeV}$. The full NLO corrections are quite large and negative around threshold, $-35 \%$, decreasing sharply to stabilise at a plateau around $\sqrt{s}=$ $600 \mathrm{GeV}$ with $-16 \%$ correction. The sharp rise and negative corrections at low energies are easily understood. They are essentially due to initial state radiation (ISR) and the behaviour of the treelevel cross section. The photon radiation reduces the effective centre-of-mass energy and therefore explains what is observed in the figure. On the other hand the genuine weak corrections, in the $G_{\mu}$ scheme, are relatively small at threshold, $-7 \%$. They however increase steadily with a correction as large as $-18 \%$ at $\sqrt{s}=1 \mathrm{TeV}$.
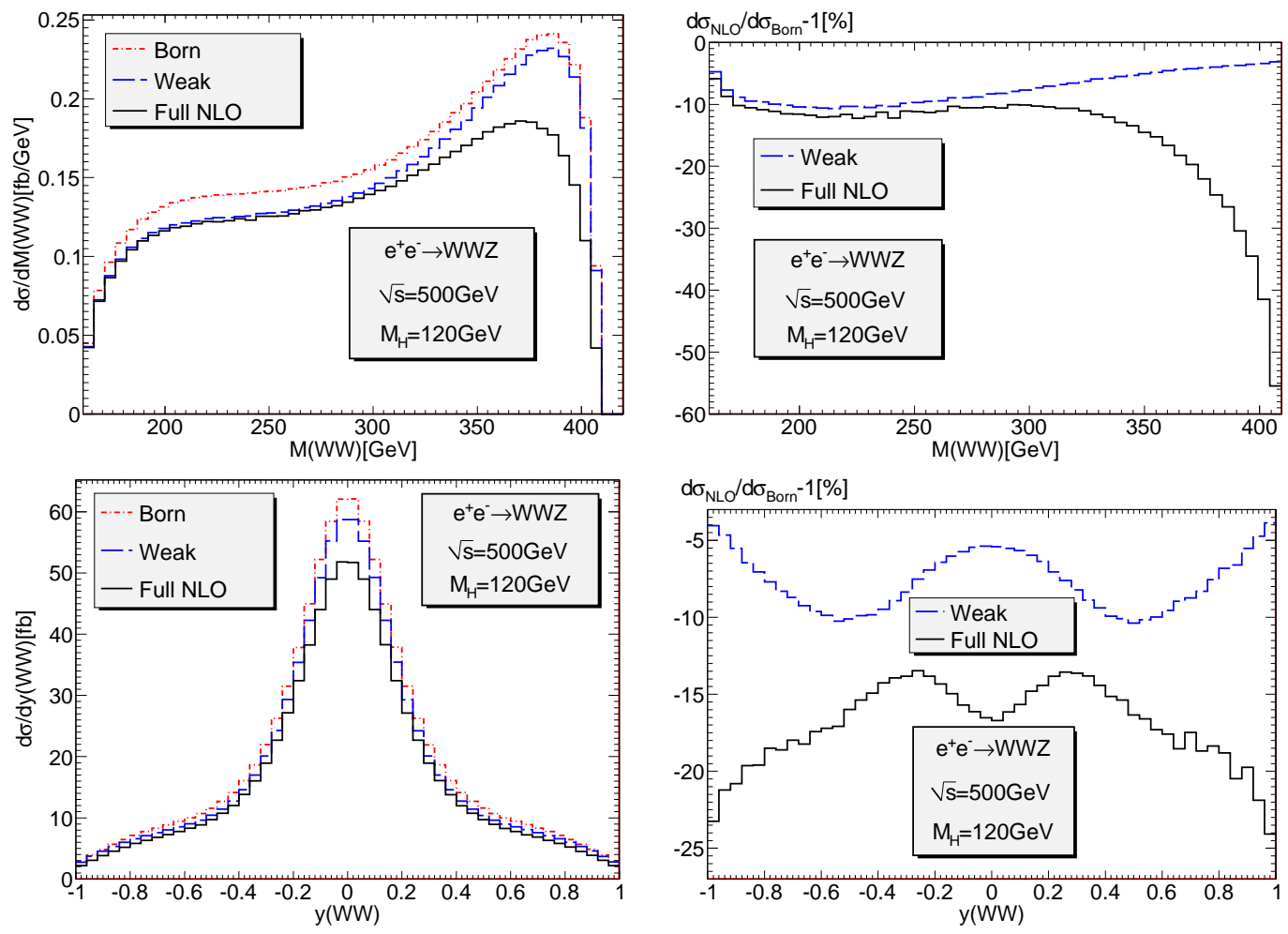

Figure 4: From top to bottom: distributions for the $W W$ invariant mass and the rapidity of the $W W$ system for $e^{+} e^{-} \rightarrow W^{+} W^{-} Z$. The panels on the left show the Born, the full NLO and the weak correction. The panels on the right show the corresponding relative (to the Born) percentage corrections.

$\underline{e^{+} e^{-} \rightarrow W^{+} W^{-} Z:}$

Compared to $Z Z Z$ production, the cross section for $e^{+} e^{-} \rightarrow W^{+} W^{-} Z$ is almost 2 orders of magnitudes larger for the same centre-of-mass energy. For example at $500 \mathrm{GeV}$ it is about $40 \mathrm{fb}$ at tree level, compared to $1 \mathrm{fb}$ for the $e^{+} e^{-} \rightarrow Z Z Z$ cross section. For an anticipated luminosity of $1 \mathrm{ab}^{-1}$, this means that the cross section should be known at the per-mil level. The behaviour of the total cross section as a function of energy resembles that of $e^{+} e^{-} \rightarrow$ ZZZ. It rises sharply once the threshold for production opens, reaches a peak before very slowly decreasing as shown in Fig. 3. 
However as already discussed the value of the peak is much larger, $\sim 50 \mathrm{fb}$ at NLO, moreover the peak is reached around $\sqrt{s}=1 \mathrm{TeV}$, much higher than in ZZZ. This explains the bulk of the NLO corrections at lower energies which are dominated by the QED correction, large and negative around threshold and smaller at higher energies. As the energy increases the weak corrections get larger reaching about $-18 \%$ at $\sqrt{s}=1.5 \mathrm{TeV}$. This is similar to the result of ZZZ production and is consistent with the behavior of double-logarithmic Sudakov corrections.

In Fig. 4 we show the distributions in the $W W$ invariant mass and the rapidity of the $W W$ system. Due to photon radiation, in the full NLO corrections some large corrections do show up at the edges of phase space. However, even after subtraction of the QED corrections the weak corrections cannot be parameterized by an overall scale factor, for all the distributions that we have studied.

\section{Conclusions}

We have presented a calculation of the full next-to-leading order correction to the processes $e^{+} e^{-} \rightarrow W^{+} W^{-} Z$ and $e^{+} e^{-} \rightarrow Z Z Z$ in the energy range of the international linear collider and for Higgs masses below the $W W$ threshold. These processes would be the successor of $e^{+} e^{-} \rightarrow$ $W^{+} W^{-}$in that they would measure the quartic couplings $W W Z Z$ and $Z Z Z Z$ which could retain residual effects of the physics of electroweak symmetry breaking. With this in mind we have subtracted the QED corrections and studied the genuine weak corrections in the $G_{\mu}$ scheme. We find that the weak corrections can be large and increase with the energy.

\section{Acknowledgments}

LDN is grateful to the organisers of the Workshop, in particular Yoshimasa Kurihara, for their invitation and financial support.

\section{References}

[1] G. Belanger and F. Boudjema, Phys. Lett. B288, 201 (1992).

[2] C. Amsler et al. [Particle Data Group], Phys. Lett. B 667, 1 (2008).

[3] http://lepewwg.web.cern.ch/LEPEWWG/

[4] T. Hahn, Comput. Phys. Commun. 140 (2001) 418, hep-ph/0012260; T. Hahn, M. Perez-Victoria, Comput. Phys. Commun. 118 (1999) 153, hep-ph/9807565.

[5] N. Baro, F. Boudjema, and A. Semenov, Phys. Rev., D78 115003 (2008), arXiv:0807.4668.

[6] A. Denner and S. Dittmaier, Nucl. Phys. B658, 175 (2003), hep-ph/0212259.

[7] F. Boudjema, A. Semenov and D. Temes, Phys. Rev. D 72, 055024 (2005), arXiv:hep-ph/0507127.

[8] F. Boudjema, L. D. Ninh, S. Hao and M. M. Weber, Phys. Rev. D 81, 073007 (2010), arXiv:0912.4234.

[9] S. Dittmaier, Nucl. Phys. B565, 69 (2000), hep-ph/9904440.

[10] S. Ji-Juan et al., Phys. Rev. D78, 016007 (2008), arXiv:0807.0669.

[11] S. Wei, M. Wen-Gan, Z. Ren-You, G. Lei and S. Mao, Phys. Lett. B 680, 321 (2009) [Erratum-ibid. 684, 281 (2010)], arXiv:0909.1064. 